\title{
FAMILIA Y EMPRESAS UN ANÁLISIS DESDE LA ANTROPOLOGÍA SOCIAL
}

\author{
Maria Josefa Santos Corral* \\ https://orcid.org/0000-0003-2696-0467 \\ Rebeca de Gortari Rabiela** \\ https://orcid.org/0000-0003-4302-622X
}

RECIBIDO: Mayo 2021 / ACEPTADO: Julio 2021 / PUBLICADO: Septiembre 2021

Como citar: Santos Corral, María; de Gortari Rabiela, Rebeca. (2021). Familia y empresas un análisis desde la antropología social. Telos: revista de Estudios Interdisciplinarios en Ciencias Sociales, 23 (3), Venezuela. (Pp. 728-746).

DOI: www.doi.org/10.36390/telos233.14

\section{RESUMEN}

El objetivo del trabajo es presentar a partir de los temas, herramientas- métodos y categorías de análisis que la antropología social, específicamente la latinoamericana, aporta en el estudio de la empresa y los empresarios. Algunos de los temas son, la incidencia de la familia en la construcción y supervivencia de las empresas, el sistema mundo en la constitución de empresas en la periferia; el análisis cultural de las elites y la relevancia de los conocimientos y la tecnología. En cuanto a las herramientas-métodos, quizá el más importante es el etnográfico que supone, como señala Geertz (1987), la descripción densa de la cultura de las empresas y empresarios, así como el análisis desde una perspectiva emic. También se destacan los aportes de la antropología de la empresa al avance de la disciplina. El método utilizado fue una revisión de la literatura sobre antropología de la empresa y los empresarios, especialmente de los trabajos de y sobre Latinoamérica. Los principales autores que refiere el trabajo son los que han hecho revisiones sobre antropología de la empresa, comenzando por el trabajo clásico de Holzberg y Giovannini (1981) y los de América Latina como los de Pérez-Lizaur (2010), Basaldúa (2005) y Urban y Kyung-Nan (2013). La contribución principal del trabajo es evidenciar la manera en que los estudios antropológicos han contribuido a explicar las particularidades socioculturales de las empresas latinoamericanas.

Palabras clave: antropología; empresas empresarios; familia; Latinoamérica.

\footnotetext{
* Investigadora del Instituto de Investigaciones Sociales de la UNAM. Email: mjsantos@sociales.unam.mx

** Investigadora del Instituto de Investigaciones Sociales de la UNAM. Email: rebeca.degortari@gmail.com
} 


\title{
Family and business from Social Anthropology
}

\begin{abstract}
The work highlights the topics, tools-methods, and categories of analysis that social anthropology, specifically Latin American, contributes to the study of the firm and entrepreneurs. Some topics are the incidence of the family in the construction and survival of companies, the world system in the constitution of periphery firms, the cultural analysis of elites, and the relevance of knowledge and technology. Regarding the tool methods, perhaps the most important is the ethnographic one, which involves the dense description, as Geertz (1987) points out, of the culture of companies and entrepreneurs and the analysis from an emic perspective. The contributions of company anthropology to the advancement of the discipline are also highlighted. The method used was a review of the literature on firms and entrepreneur's anthropology, especially those works of and about Latin America. The main authors referred to in work are those who have made reviews on the anthropology of the company, starting with the classic work of Holzberg and Giovannini (1981) and those of Latin America, such as those of Pérez-Lizaur (2010), Basaldúa (2005) and Urban y Koh (2013). The main contribution of the work is to show how anthropological studies have contributed to explaining the socio-cultural particularities of Latin American companies.
\end{abstract}

Keywords: anthropology, business enterprises; family; Latin America.

\section{Introducción}

El propósito de este trabajo es destacar los temas, herramientas- métodos y categorías de análisis que la antropología social, específicamente la que se hace desde Latinoamérica, aporta para el estudio de la empresa y los empresarios. Se parte de que la antropología industrial o de la empresa, es aquella rama especializada de la disciplina que establece como unidad de observación el entorno industrial empresarial (Roca i Girona, 2001). La industria agrupa firmas de negocios, fábricas, talleres, almacenes, agronegocios, minas, cadenas comerciales, pequeños comercios que producen y/o bienes y servicios en nuestras sociedades (Gamst, 1977).

En estos espacios en tanto organizaciones, se establecen patrones de comportamiento, símbolos, rutas de comunicación y arreglos para la interacción entre actores que laboran en las industrias y aquellos que se ubican en su entorno regional y global. A ello, por una parte, hay que agregar las subculturas a las que se encuentran asociados los trabajadores; pensemos por ejemplo en los jornaleros agrícolas, los mineros o los obreros industriales. Por otra, las referencias asociadas a las cadenas globales de proveeduría en las que muchas veces se encuentran inmersas estas empresas y, por último, los marcos legislativos, económicos y relacionales de los lugares donde se ubican las empresas.

En este sentido, los trabajos construidos desde la antropología o que retoman el enfoque de esta ciencia contribuyen, en principio, a esclarecer las diferencias derivadas de la superposición de referencias $y$, en ocasiones, proporcionan argumentos para entender y resolver algunos de los problemas que se plantean entre la administración y la fuerza de trabajo. La importancia del presente trabajo radica en retomar cuáles son las particularidades de las 
Familia y empresas un análisis desde la antropología social.

referencias latinoamericanas en la construcción de las empresas y en la labor de emprendimiento.

Los trabajos de antropología de la empresa y de los empresarios datan de la década de 1970, cuando desde la etnología se comenzaron a estudiar las propias sociedades urbanas tanto en EU como en Canadá (Durrenberger, 2007). Los empresarios, por su parte, también han sido objeto de estudios antropológicos desde la perspectiva del análisis de las elites. Cerutti (2003) señala que estos trabajos, construidos bajo la perspectiva de la historia socioeconómica, tuvieron cierto auge desde la época de los 80.

Desde la antropología latinoamericana son pioneros los trabajos de Lomnitz y PérezLizaur (1993) en los que la discusión se centraba en la manera en que las relaciones sociales, especialmente aquellas basadas en el parentesco se constituían en un mecanismo muy eficaz para obtener los recursos materiales, y sociales que se necesitan para constituir una empresa. A los que le siguieron otros como el análisis cultural de las elites Baradó y Veccioli (2009); temas como la incidencia del sistema mundo en la economía de las familias empresarias de la periferia (Azevedo, 2012) y, la relevancia de los conocimientos y tecnología, entre otros. En cuanto a las herramientas-métodos, rescatados para estos trabajos, quizá el más importante es el etnográfico que supone la descripción densa (Geertz, 1987), de la cultura de las empresas y empresarios, para entender los recursos y los conocimientos que ensamblan y movilizan en su camino para emprender (Reygadas, 2000; Santos y de Gortari, 2016). El método se encuentra ligado a la categoría cultura y al establecimiento de redes; categorías ambas construidas y trabajadas desde la antropología.

El trabajo está construido en tres apartados. En el primero se presentan los aportes iniciales de la antropología a los estudios de empresas y empresarios y la manera en que comenzó a gestarse y legitimarse una rama de la disciplina, la de la antropología de la empresa. En el segundo, se revisan los trabajos que antropólogos latinoamericanos han hecho sobre el tema de familia y empresa, donde la familia y las redes sociales constituyen un recurso muy valioso para la creación y crecimiento de las empresas. El tercer apartado, muestra los aportes de la antropología, especialmente la que se hace en Latinoamérica, a los estudios de empresas y empresarios, al tiempo en que se rescatan algunas de las contribuciones de los trabajos de empresas y empresarios para enriquecer el trabajo antropológico. El artículo cierra con unas conclusiones acerca de las aportaciones, temas, herramientas y métodos, así como de categorías de análisis de la antropología, de igual manera que se señalan algunos retos.

El método seguido para construirlo fue la revisión de la literatura sobre antropología de empresas y empresarios. El criterio de búsqueda fue que los trabajos recuperaran el método etnográfico o ciertas categorías de análisis de la antropología en el estudio de empresas y empresarios. En este sentido la búsqueda comenzó recuperando el trabajo clásico de revisión de Holzberg y Giovannini editado en 1981 sobre antropología de la industria y se recuperaron trabajos de autores latinoamericanos publicados en revistas como Nueva Antropología, otras prestigiosas revistas latinoamericanas, así como libros sobre la temática de empresas y empresarios en el periodo que va de 1980 a 2020. Dentro de la revisión se excluyeron aproximadamente 20 trabajos que no referían específicamente los aportes etnográficos 0 no recuperaban las categorías como las de cultura, familia y/o elites, además de los que están planteados desde la antropología considerando a la empresa y a los empresarios como nuevos sujetos de estudio. 


\section{De cómo la antropología se comienza a interesar por las empresas y las familias de los empresarios.}

La antropología comienza como disciplina analizando el entorno social, ideológico y tecnológico, esto es la cultura (White, 1964) de comunidades corporadas y cerradas relativamente autocontenidas, lo que facilita el acercamiento desde una perspectiva holística. A manera de ejemplo se pueden citar los trabajos de las monografías clásicas tanto las de los profesionales británicos como, las de los norteamericanos y franceses interesados en relativizar los comportamientos de los grupos sociales con los que trabajaban. Este método de aislar sociedades resulto muy atractivo para los psicólogos sociales que lo siguieron para sus trabajos de y sobre empresas. Quizá el más conocido es el de Elton Mayo realizado entre 1929 y 1932 en la Western Electric Company, situada en Chicago, donde retomo la metodología etnográfica, (especialmente la observación in situ que supone el trabajo de campo) de los estudios de Malinowski, para plantear una investigación sobre la relación entre la fatiga y el comportamiento en un grupo de trabajadores.

En el estudio se encontró que, el proceso de producción podría modificarse no solo a través de la tecnología, sino también, cambiando la estructura organizacional informal y / 0 formal de los grupos de trabajo (Holzberg y Giovannini, 1981). Los trabajos del taller de borneras de la Western Electric Company llevaron a la fundación de la escuela de las Relaciones Humanas, desde cuyo enfoque, se caracterizó a la empresa como una pequeña sociedad en sí misma, con una "cultura propia" consistente en rutinas, patrones, regularidades, métodos de documentación, símbolos, etc. en la que se podía trazar un mapa de la estructura social, para delinear tanto los rasgos formales como los informales de la organización.

Se asumió que los conflictos y tensiones en las relaciones humanas afectaban negativamente a la producción, porque eran manifestaciones de rupturas en el equilibrio de la empresa. Por lo tanto, para volver a un estado de armonía, que fomentara un buen ánimo entre los trabajadores y que contribuyera a lograr una producción eficiente, era necesario hacer reajustes en el sistema social de interacción pautada, cambiando rutinas de trabajo que facilitaran la interacción, (Holzberg y Giovannini, 1981).

Se abundó en esta investigación que podría considerarse como la primera en la que los comportamientos industriales son observados desde la lupa de la antropología, por dos razones. En principio, porque la intervención antropológica ocurre en el laboratorio de otra disciplina (la psicología), lo que marca el comienzo de la interdisciplinariedad, donde la contribución de la antropología estuvo centrada en desmadejar el atolladero en el que había quedado sumida la investigación del taller, evidenciando la función e influencia de la organización informal de los trabajadores en la productividad; asunto que se constituyó en el mayor aporte de la Escuela de Relaciones Humanas, (Roca i Girona, 2001). En segundo lugar, porque con la metodología etnográfica de Malinowski, se filtró también, el análisis de la realidad de la planta industrial desde una perspectiva centrada en el actor (emic) donde factores como la reciprocidad, la cooperación y también la competencia (Bueno, 2016) controlan las jerarquías y los recursos presentes en los grupos informales. Es así como, tanto el análisis interdisciplinar como la perspectiva del actor, han sido aportaciones importantes de la antropología para los estudios de la empresa. 
Familia y empresas un análisis desde la antropología social.

Además, y como lo señala Roca i Girona en sus trabajos de 1999 y 2001, el enfoque de esta escuela abrió oportunidades para el campo de la antropología industrial. Hasta 1950, el potencial de la disciplina para los estudios industriales quedo evidenciado en dos tipos de trabajos. En primer lugar, aquellos que analizan los fenómenos laborales, aunque en este periodo, estuvieran separados del medio social en el que se producían. En segundo, y muy vinculado con lo anterior, los estudios centrados en la descripción etnográfica de las culturas de trabajo, donde se hacía énfasis tanto en los procesos tecnológicos como en las relaciones humanas.

Para la década de 1950 y 60 la investigación industrial-empresarial de la antropología norteamericana comenzó a recuperar otras tradiciones como la de la Escuela de Manchester, con la influencia de Gluckman, que dio lugar a una serie de investigaciones sobre organizaciones industriales, en las que se evidenciaron los conflictos y las contradicciones de la estructura organizacional y de los procesos tecnológicos, y en las que además, se planteó el trabajo de campo desde la observación participante (Roca i Girona,1999). Un buen ejemplo de ello es el trabajo de Lupton (1963) en la Wye Garment Company, que rescata la manera en que los comportamientos de los trabajadores están normados por las referencias de la empresa.

En las siguientes décadas, se superaría el modelo de la fábrica como un sistema cerrado, contextualizando la situación del taller en estructuras sociales más amplias, de manera que las "sociedades complejas" comenzaron a ser el objeto de análisis (Roca i Girona,1999). Ejemplo de ello son los trabajos de Burawoy (1979) sobre el papel que tuvo el Estado de bienestar norteamericano en las reivindicaciones obreras, y el de Gamst publicado en 1977 que analiza la cultura laboral desde lo que el autor caracterizó como un orden social más amplio, (Bueno, 2016).

En el mismo periodo, además del contexto social en el que se ubican las empresas, algunos de los trabajos retoman otro de los pilares de la antropología: el método comparativo, lo que permitió describir y explicar las similitudes subyacentes que caracterizaban entornos industriales aparentemente diversos. Tal es el caso del trabajo de Lucas que a finales de los sesenta analiza, para estudiar una sola industria, los patrones demográficos, económicos y socioculturales que son comunes a tres ciudades canadienses donde se localizan sus talleres (Lucas 1971 citado en Holzberg y Giovannini, 1981).

En los años recientes, el análisis de la globalización ha llamado poderosamente la atención de las ciencias sociales incluyendo a la antropología. Uno de los aspectos más trabajados desde la disciplina, ha sido la incidencia de las referencias globales en las culturas empresariales locales, bien sea porque las empresas analizadas forman parte de grupos 0 holdings trasnacionales, porque están integradas a las cadenas de proveeduría de estos grupos, o simplemente porque incluso en los nichos de mercado regionales donde venden sus productos, compiten con las empresas globales.

Las referencias globales inciden en los procesos tecnológicos, estructuras organizacionales, y en la construcción misma de los marcos que guian sus acciones. Un trabajo que muestra la manera en que la globalización influye en las culturas industriales locales es el de Reygadas (2000), quien a partir de descripciones concretas de globalización a la que se han visto sometidos los obreros de maquiladoras de México y Guatemala, analiza la intersección entre las relaciones de poder y los procesos interculturales en el trabajo, distinguiendo entre tres fenómenos: la globalización que implica procesos generales de mundialización, poco 
institucionalizados; el transnacionalismo, donde hace referencia a las relaciones de hegemonía entre los diferentes Estados nación y, la relocalización, centrada en los esfuerzos de los agentes productivos locales (en este caso trabajadores, empleados y gerentes guatemaltecos y mexicanos). Todo ello para mostrar la manera en que los actores adaptan y transforman sus culturas de trabajo con el fin de controlar mejor el funcionamiento de las maquiladoras.

En suma, se puede señalar que los estudios de antropología de la industria o de la empresa si bien parten del uso del método etnográfico, al adoptar éste permean las categorías, los métodos y los presupuestos con los que trabaja la antropología. Esto es, se filtran categorías como: cultura, redes, el método comparativo, el relativismo cultural y, sobre todo, la perspectiva emic. Además, muchos de los trabajos de estos primeros antropólogos contribuyeron a sacar a la antropología del análisis de las sociedades "simples" (grupos tribales y comunidades indígenas en Latinoamérica), para acercarlos a los estudios de sociedades complejas, más cercanos a las realidades de los investigadores.

En cuanto a los estudios de empresarios, como se refirió arriba, comenzaron desde el trabajo que los antropólogos podrían hacer para explicar el comportamiento de las elites. En este sentido, las herramientas antropológicas contribuyen al análisis de las prácticas y referencias de quienes ocupan posiciones de poder. Lo anterior plantea la necesidad de atender los desafíos teórico-metodológicos, que parten de que la antropología es una ciencia social, cuyos sujetos de estudio han sido los subalternos marginales y los desposeídos. Las nuevas formas de trabajo implican consideraciones en los planos epistemológico, ético, y en el aspecto metodológico, que comienzan con la manera en que se puede acceder a estos grupos, donde el investigador es ahora el subordinado.

En el plano epistémico, se tiene que considerar que el capital social y cultural de los colectivos analizados les permite no solo leer, sino también, cuestionar lo que se escribe sobre ellos. En el aspecto ético, el desafío para el antropólogo consiste en encontrar la manera de darles voz a los sujetos sin transformarse en un "vocero del grupo", (Gessaghi, 2011). Además, se debe considerar que trabajar en un entorno empresarial podría implicar una relación contractual en la que un empleador (el empresario, o algún integrante de la empresa) contrata los servicios de un profesional, en este caso el antropólogo, para mejorar su empresa y con ello los beneficios que se obtienen. Así, en la medida que los intereses del capital y los del trabajo no son coincidentes, colaborar con la empresa puede significar ir en contra de los trabajadores, quienes por su posición subordinada resultan hipotéticamente más próximos a los antropólogos (Roca i Girona, 2001).

En Latinoamérica, desde principios de los 80 , los estudios empresariales podrían agruparse dentro de lo que Cerutti (2003) caracterizó como una rama de la historia económica, fueron aceptados en "los márgenes de algunas ciencias sociales" como la historia y la sociología. Algunos de los temas considerados en estos trabajos son: Estado-empresarios, historia empresarial, historia regional y empresarial, estudios de los procesos de trabajo y biográficos, empresarios y género, hombres de negocios en México y Colombia y procesos históricos de los empresarios latinoamericanos donde se analiza la correlación entre el desempeño de ciertos empresarios exitosos, y sus familias como recursos que permiten consolidar y generar lazos económicos o políticos, (Cerutti, 2003; Hernández, 2013).

El último tema del presente apartado es el vínculo entre familia, empresarios y empresa. Al respecto se señala, que el análisis de la familia ha estado presente desde los 
Familia y empresas un análisis desde la antropología social.

primeros estudios antropológicos sobre "sociedades simples", donde los investigadores tuvieron que entender y explicar los complejos sistemas de parentesco de las sociedades que analizaban. Al respecto destaca el trabajo temprano de Ramírez Carrillo (1992) sobre los empresarios libaneses en el sureste de México. Por su parte, Nader (1972) refiere que, la antropología está interesada en los determinantes de los patrones familiares de los empresarios (más que de los pobres 0 de los ricos) pues con ello, si se analiza de manera comparada, tal vez se logren comprender las fuerzas que generan excesiva pobreza o de los determinantes culturales de transformación del grupo.

Pérez-Lizaur en su trabajo de 2010, define la empresa familiar como "una organización controlada y operada por la familia, donde las estructuras y relaciones típicas de ésta, se trasladan y perpetúan en las organizaciones de trabajo" (Pérez-Lizaur, 2010:21). La misma autora señala que, muchas veces el modelo de empresa familiar coincide con el de familia de las sociedades donde estas se encuentran ubicadas. Además, analizar la estructura y funcionamiento de las familias es relevante, porque es a partir de las referencias culturales locales presentes en las familias de los empresarios y de los trabajadores, como la localidad permea en la cultura de las empresas.

Por otro lado, y como se verá con mayor amplitud en el siguiente apartado, en el ámbito de empresa y familia, una de las preguntas que la antropología ha tratado de resolver es hasta dónde la participación laboral de los integrantes de la familia y las relaciones que se establecen entre ellos en el espacio de la empresa, constituyen una forma de producción que permite asegurar la reproducción, tanto de la unidad económica como del consumo y el abastecimiento de la unidad familiar (Román, 2011). En el siguiente apartado, se presentan los estudios y las aportaciones sobre este tema en los trabajos de los antropólogos latinoamericanos.

\section{Los estudios de familia y empresa en América Latina, su origen y algunas contribuciones}

Los estudios de familia y empresa en Latinoamérica parten de considerar que la familia es un actor central en la determinación de las condiciones sociales, económicas y políticas de la región. Así lo señala el número especial sobre familias latinoamericanas publicado por el Journal of Family History en 1985, donde los editores sostienen que esta centralidad se vincula con varios temas: el rol empresarial de la familia, la familia y la política en el periodo de consolidación del Estado nacional, los lazos de parentesco y la estructuración del espacio social urbano (Jelín y Paz, 1991: 2).

En efecto, en la mayoría de los trabajos de América Latina sobre empresas, emprendedores y familia, están siempre presentes los recursos sociales, materiales, políticos y de conocimientos con lo que las familias cuentan para emprender y mantener talleres, industrias y comercios. La actividad emprendedora requiere del manejo de recursos materiales, sociales y en Latinoamérica muchas veces también políticos, y la familia (nuclear y/o extensa) es el vehículo para conseguirlos. De tal suerte que, "el empresario y la empresa forman una red cardinal compuesta por relaciones sociales, recursos económicos, conocimientos técnicos y manejo del mercado, donde aparece la idea de una trayectoria desde la composición de empresa familiar" (Basaldúa, 2005:7). El mismo autor refiere el trabajo de Pérez-Lizaur (1994) quien considera que, el empresario es un actor social que aprovecha oportunidades del entorno y para ello crea y organiza empresas con los recursos a su alcance, utilizando y reordenando las relaciones sociales, (Pérez-Lizaur citado en Basaldúa, 2005). 
Los primeros trabajos latinoamericanos sobre empresa y familia comenzaron analizando el papel que juega ésta en la articulación de los negocios, donde el aporte de la antropología se encuentra en señalar asuntos como la manera en que los integrantes de las familias son al mismo tiempo propietarios y trabajadores de los espacios productivos, en los que la participación laboral de los miembros en la empresa, taller artesanal o comercio y las relaciones que se establecen entre ellos, constituyen una forma de producción que permite asegurar la reproducción de la unidad doméstica, el consumo y el abastecimiento de la unidad familiar (Román, 2011).

Posteriormente, estudios como los citados por Reguera (2009), Hernández Romo (2017) y los de Lomnitz y Pérez-Lizaur (1993) destacan el papel de los empresarios y sus familias, para insertarse en contextos institucionales y manejar relaciones políticas que permitan construir y hacer crecer sus empresas. Al considerar también las estrategias (alianzas matrimoniales y prácticas de sucesión) para mantener el emprendimiento en el tiempo y, de acuerdo con la coyuntura, apelar al ahorro 0 al crédito para decidir las inversiones. Para analizar lo anterior es fundamental relacionar la trayectoria singular de una empresa 0 empresario, con la dinámica del contexto y con las transformaciones del sector económico, que permite la acumulación de bienes materiales y simbólicos (Reguera, 2009).

Este apartado se centra en dos temas tratados por los estudios antropológicos de empresa, empresario y familia. Aquellos que se refieren al vínculo unidad doméstica-familia y empresa, y los que destacan el papel de la familia que en tanto red primaria se constituye en una fuente de recursos sociales, económicos y de conocimiento para la integración y consolidación de las empresas. En este último, se hace una breve referencia al papel que juegan las redes familiares en la integración de espacios globales para el desarrollo de las empresas.

\section{La empresa como alternativa de reproducción de la unidad doméstica}

Para comenzar a analizar esta relación es importante señalar la contribución de las MiPymes a la economía Latinoamericana en donde emprender y establecer una empresa se ha convertido en una práctica como alternativa de reproducción de la unidad doméstica, ello sobre todo si se considera la estructura económica que priva en la mayoría de los países latinoamericanos. De acuerdo con la COEC, generan la mitad de los puestos de trabajo; son el $90 \%$ del tejido empresarial y suponen el $28 \%$ del PIB. El mismo documento señala que éstas no reciben la atención necesaria de las políticas públicas de la región (COEC, 2018). Los porcentajes anteriores varían de acuerdo con el país. Por ejemplo, en Argentina de las 856.300 empresas que se tenían registradas para $2016^{1}, 83 \%$ eran microempresas; $16,8 \%$, pymes, y solo $0,2 \%$ grandes compañías. Además, las firmas de 1 hasta 200 empleados representan el $66 \%$ del empleo formal privado del país, según datos de la Secretaría de Transformación Productiva. Mientras que, en México, según datos del INEGI, las MiPymes generan el $72 \%$ del empleo formal nacional. Los datos censales también incluyen el tipo y sector de las empresas que entran en esta categoría y que van de las pequeñas tiendas de abarrotes a las empresas con alto contenido tecnológico. Lo que no se suele registrar en estos censos es el papel de que juega la familia para sostener financiera y socialmente estos emprendimientos.

\footnotetext{
${ }^{1}$ Este es el último dato registrado, con la pandemia los números variaron pues muchos de estos negocios cerraron.
} 
Familia y empresas un análisis desde la antropología social.

Algunos de los trabajos antropológicos en este rubro, equiparan la familia con la unidad doméstica ${ }^{2}$, donde sus integrantes aportan relaciones, recursos y en ocasiones trabajo para que el taller, el comercio o la fábrica industrial prospere. Asunto de gran relevancia si se atiende a los datos con los que se inició este apartado. Por ejemplo, García y Oliveira plantean la vinculación entre dos organizaciones, la unidad doméstica y la unidad económica, en la medida en que la familia, en tanto institución flexible, va readaptando sus formas, funciones, papeles y resistencias a los cambios sociales y económicos. Así la unidad doméstica empresarial se convierte en "un ámbito de interacción y organización de los procesos de reproducción cotidiana y generacional de los individuos vinculados o no por relaciones de parentesco. Allí se crean y recrean relaciones de autoridad, solidaridad y conflictos de intercambio y poder" (García y Oliveira, 1996: 15 citado en Román, 2011).

Román también destaca el papel reproductor de la familia y retoma para ello el trabajo de Jelín y Paz que señalan que la familia como unidad de procreación y sexualidad, ha comenzado a ser tratada como un sistema de vínculos afectivos que se van superponiendo más allá de la convivencia, por ello debería de ser pensada como un "techo abierto", donde las relaciones entre madre-hijo(s); padre-hijo(s), hermanos y otros parientes, constituyen los verdaderos componentes del sistema (Jelín y Paz, 1984: 126 citado en Román, 2011).

Como se señaló arriba, la empresa es un espacio que permite brindar oportunidades laborales para los integrantes de la familia y con ello contribuir a la reproducción de una o varias unidades domésticas. En ocasiones, la idea de emprender comienza después de la jubilación y/o despido de uno de los familiares y la primera etapa de la empresa, suele sostenerse gracias a las aportaciones económicas de algún otro integrante de la familia, bien sea para apuntalar el negocio, o bien para no distraer los recursos invertidos en éste en mantener la unidad doméstica.

Al mismo tiempo, se puede contar con el subsidio de la mano de obra familiar bajo un esquema en el que las tareas domésticas y de reproducción son claramente indiferenciadas. Román considera que, en este tipo de unidades, los procesos que ocurren en el interior del hogar se encuentran fuertemente vinculados con la organización, tanto de la vida familiar en distintos ámbitos, como del trabajo de sus integrantes lo que ocurre especialmente en tres tipos de empresas: artesanales de tipo industrial, industrias capitalistas a domicilio y talleres industriales o manufactureros. Donde la empresa mantiene un vínculo indisoluble con el hogar, y la familia; es simultáneamente la dirigente del negocio y la coordinadora del hogar, por lo que la organización de ambas unidades está en manos de la familia, que debe combinar sus responsabilidades (Román 2011).

Por último, de acuerdo con Pérez-Lizaur, los estudios que retoman desde una perspectiva culturalista el papel de la unidad doméstica en la construcción y consolidación de las empresas se vinculan también a los procesos de desarrollo regional (Pérez-Lizaur, 2010). Así lo muestran trabajos en los que se destaca la manera en que se tejen relaciones de parentesco y vecindad en la constitución de pueblos-empresas. Concepto que describe aquellos espacios sociales cuyo surgimiento está asociado a la creación de una industria, y cuyas dinámicas son reguladas, por los empresarios que controlan los procesos de construcción y transformación regional. Donde el ascenso social vinculado a la actividad empresarial se

\footnotetext{
${ }^{2}$ La unidad doméstica, se define como "un lugar geométrico o esfera social compuesta de una serie de personas que comparten un lugar común donde vivir y un presupuesto" (Benería y Roldán, 1987: 140). Es un espacio para que provea de los recursos materiales necesarios para mantener y criar a sus miembros.
} 
encuentra en los recursos de las organizaciones primarias tradicionales como la familia extensa; empresas familiares que son las que imprimen a la localidad gran dinamismo (Cortés, 2013).

\section{La familia red proveedora de recursos socio-materiales}

La antropología comenzó a trabajar con el análisis de redes sociales desde 1960, a partir de los trabajos que, desde la Escuela de Manchester, conducidos por Gluckman, se realizaron en zonas urbanas de Zambia. En éstos, se caracterizó a la red social como una estructura que puede ilustrarse por medio de uno o varios grafos, en los cuales los nodos representan individuos u otras redes, denominados "actores"; y las aristas refieren las relaciones entre ellos. Las relaciones al interior de la red propician operaciones de intercambio, y a partir de ellas, pueden analizarse elementos como: anclaje, accesibilidad, densidad y rango (Santos y de Gortari, 2016).

Autores como Hung (2006), resaltan dos tipos de redes que inciden en el desarrollo de nuevos emprendimientos: las interpersonales y las interorganizacionales. Las primeras, constituidas por los sistemas sociales en los cuales el empresario se encuentra inmerso: familia, amigos, colegas y sus contactos de negocios, permiten el análisis del ambiente social donde se mueven los emprendedores. Las segundas, muestran la manera en que el emprendimiento establecido se relaciona con otras organizaciones en su operación y crecimiento, (Hung, 2006 citado en Santos y de Gortari, 2016).

En Latinoamérica, las redes de empresarios han generado organizaciones y grupos a partir de núcleos como las familias (Basaldúa, 2005). Estas redes se constituyen en espacios en los que es posible acumular recursos que permiten a los actores ubicados en ellas, competir en los mercados nacionales e incluso en los globales. En las redes sociales se acumulan también, saberes y conocimientos que posibilitan la integración y consolidación de empresas. Las redes refuerzan la identidad cultural, conservan los recursos naturales y ayudan a preservar tradiciones locales y regionales (de Gortari y Santos 2010).

A diferencia de los trabajos sobre unidad doméstica, familia y empresa, los estudios empresariales latinoamericanos que retoman la perspectiva de las redes, mayoritariamente se plantean desde la construcción y reproducción de las élites empresariales presentes en la región. Un trabajo pionero en este sentido es el de Larissa Lomnitz y Marisol Pérez-Lizaur (1993), en el que las autoras analizan una familia de la élite de la Ciudad de México a lo largo de tres generaciones.

El trabajo se construye desde una combinación de enfoques macro-micro donde, el análisis de redes sociales que descansan en estructuras de parentesco se entreteje con las vicisitudes sociales y políticas que vive México a lo largo de la historia de la familia. González y Basaldúa apuntan que metodológicamente las autoras incluyen varios niveles de análisis: la perspectiva individualista que parte de la observación del contexto en el que se desarrollan los sujetos en un período de tiempo específico y, la composición de los grupos, desde una perspectiva estructuralista. Para ello, describen la manera en que varios de los integrantes de una familia establecen redes empresariales interdependientes, en donde se puede seguir el entramado de las relaciones individuales y políticas (González y Basaldúa, 2007).

En el mismo trabajo de revisión, González y Basaldúa recuperan otros estudios sobre empresarios, redes y élites en los que se enfatiza el papel de las redes para establecer ligas con proveedores, clientes y con el Estado, mostrando el rol que juega el intercambio de información 
Familia y empresas un análisis desde la antropología social.

en el interior de la red, en las interacciones y transacciones que tienen como propósito lograr que se consoliden los objetivos de las empresas, (González y Basaldúa, 2007).

Mas recientemente, se encuentran los trabajos de Cárdenas (2016) y Castellani y Heredia, (2020), que muestran la relación entre las redes y la consolidación de elites empresariales supranacionales. En su trabajo, Cárdenas parte de la premisa de que el análisis de las redes de interlocking directorates y de propiedad ofrece la oportunidad de comprender la cohesión de las elites empresariales. El estudio centrado en tres países latinoamericanos México, Chile y Brasil analiza la manera en que las redes tejidas entre personas y organizaciones que dirigen y controlan las grandes empresas, generan estructuras relacionales que condicionan el comportamiento de las elites y el entorno económico en el que éstas se desenvuelven. La manera en que se enredan las grandes empresas y sus elites incide en los préstamos adquiridos, el financiamiento de los partidos políticos y el apoyo a organizaciones no gubernamentales. Afecta también el tipo de capitalismo que emerge en un país e incluso en la forma en que se globalizan sus economías (Cárdenas, 2016).

Por su parte Castellani y Heredia (2020) a partir del seguimiento de las 50 primeras compañías argentinas y sus presidentes, en el periodo 1976-2015, analizan la cohesión generada en las redes que vinculan a los actores dominantes, y como ello incide en el proceso de renovación o consolidación de las posiciones adquiridas en un contexto de inestabilidad económica. El trabajo resalta dos hallazgos: un notable nivel de renovación empresarial y el recorte de alguno de los beneficios gubernamentales a quienes ocupan posiciones de élite.

A manera de resumen, en este apartado se mostró la importancia de las familias y sus redes para la clase empresarial latinoamericana, señalando la contribución específica de la antropología para el análisis de estos grupos sociales. Para cerrar el trabajo, a continuación, se presenta la forma en que las categorías y métodos de la antropología han contribuido a los estudios de empresas y empresarios, pero también, la manera en que estos trabajos han contribuido a enriquecer las categorías y métodos antropológicos.

\section{Aportaciones y retos de la antropología para los estudios de empresa y empresarios.}

En primer lugar, se exponen los aportes de la antropología para los estudios de empresas y empresarios a nivel global y, específicamente para Latinoamérica. En segundo, se hace una reflexión de hasta dónde los trabajos sobre empresas y empresarios han contribuido a enriquecer las categorías y los métodos de esta disciplina.

En principio, se puede decir que el enfoque de la antropología ayuda a las empresas en la gestión de cuestiones como las diferencias culturales, las étnicas y en algunos casos, actúa como articulador entre la administración y la fuerza de trabajo. Por otro lado, a partir del estudio de las corporaciones, la antropología ha contribuido a replantear y resolver, junto con otras diciplinas, problemas de las empresas y con ello, ha desarrollado nuevos enfoques tales como: la antropología organizacional esto es, el estudio de organizaciones complejas que incluye el componente cultural, los procesos de trabajo y los cambios de directivos; la antropología de marketing y comportamiento y, la antropología de diseño (productos y servicios). Recientemente los trabajos antropológicos han incursionado en áreas de inteligencia competitiva, negocios internacionales y de transferencia de tecnología y conocimiento (Lima, 2009).

En los estudios sobre empresarios, la antropología ha contribuido a evidenciar el contexto histórico-institucional que los sostiene, vinculando la trayectoria específica de una 
empresa, de un empresario, o de un grupo de empresarios con la dinámica del contexto y con las transformaciones del sector económico y de los procesos sociales. Esto cobra importancia porque la actividad de emprender supone haber acumulado previamente capital social, económico y en ocasiones simbólico (Reguera,2009).

A continuación, se presentan los aportes de las herramientas, métodos y categorías de análisis de la antropología al estudio de empresas y empresarios, y, la manera en que los temas de empresas y empresarios han enriquecido el quehacer de la disciplina.

\section{Los aportes: el método etnográfico y las categorías de cultura y elite}

Sin duda el primer gran aporte de la antropología a los estudios de empresas y empresarios es el trabajo etnográfico, bajo el que se rescatan las formas en que el informante analiza, interpreta y explica la realidad, considerando el contexto de los grupos de interés a los que se encuentra adscrito (Peña, 2008). Así, y aun cuando parece superado, el trabajo de campo derivado de la antropología clásica ha permitido resolver los tropiezos que se presentan al realizar entrevistas en campo, en el levantamiento de la información, e incluso, para decidir sobre la postura que el antropólogo debe tomar cuando se niega o se brinda información. Con ello, las herramientas derivadas del trabajo de campo contribuyen a trazar la ruta del análisis posterior, en la elaboración de aspectos teóricos y en la presentación de los resultados del estudio; en el sentido en que, los datos recabados permiten encontrar el modelo de articulación social, observado a nivel micro-social (González y Basaldúa, 2007).

La investigación etnográfica dentro de las corporaciones, parte de que éstas son grupos sociales, caracterizados por varios de los elementos culturales que los antropólogos han documentado para diversas sociedades a lo largo del mundo, operan como sociedades de pequeña escala y en algunos casos de gran escala, con sus propios mitos internos, rituales, creencias, normas y prácticas. Al mismo tiempo, la corporación empresarial es un tipo distintivo de agrupación social, en la medida en que está orientada a la obtención de beneficios económicos, por encima de otros objetivos.

A partir del análisis de las actividades empresariales en relación con la sociedad, el trabajo etnográfico ha documentado asuntos como los efectos de las actividades de corporaciones transnacionales, principalmente extractivas en poblaciones locales del Cuarto y Tercer Mundo, evidenciando las respuestas políticas de las comunidades. Otras investigaciones apuntan a describir asuntos vinculados con la cultura como el desarrollo de mitos o la formación de clanes, en y alrededor de las empresas. En este sentido, la información construida desde la antropología puede jugar un papel importante en el cambio de énfasis en las funciones sociales de la corporación que benefician a la sociedad, además de llamar la atención a sobre sus efectos nocivos (Urban y Koh, 2013). La etnografía muestra la manera en que las empresas se constituyen en actores poderosos en la escena mundial, lo que incide no solo en las personas que trabajan en ellas, sino también, en las culturas locales, así como en el medio ambiente.

El trabajo etnográfico planteado desde la categoría de cultura, permite analizar la forma en que las prácticas laborales pueden reorganizar las relaciones de género, los patrones familiares y suscitar diversas respuestas culturales que impactan en el entorno donde operan. Además, los bienes y servicios producidos por las empresas también difunden valores culturales, en ocasiones distintos a los de la sociedad que las reciben. Así, quizá una de las mayores 
Familia y empresas un análisis desde la antropología social.

contribuciones de los estudios etnográficos sobre los efectos de las empresas en el lugar de trabajo es la documentación de la interacción y el impacto de la corporación en la cultura exterior.

A partir de una revisión de la literatura antropológica Urban y Koh sugieren dos formas distintas desde las que los etnógrafos se han acercado a la corporación empresarial como formación cultural: aquella que analiza los efectos de las empresas en trabajadores, comunidades, consumidores y medio ambiente; $y$ la que se centra en el funcionamiento interno de las corporaciones como sociedades a pequeña escala y/o a gran escala. Ambos acercamientos posicionan a la disciplina para contribuir al avance de la caracterización de la empresa, y también a proponer cuestiones prácticas, no solo para el seguimiento de impactos dañinos, sino también, brindando sugerencias para mejorar beneficios sociales, (Urban y Koh, 2013).

Por otro lado, los mismos autores destacan que la investigación etnográfica ha considerado poco las diferencias de las empresas atribuibles al tipo de propiedad, estatal versus privada; tema que había sido un foco de atención en los estudios de Burawoy sobre las fábricas bajo el comunismo y el capitalismo. Tampoco se han examinado las variaciones atribuibles a tenencias de acciones difusas versus concentradas 0 , a la representación obligatoria de sindicatos en juntas directivas, asuntos que si han sido estudiados por los politólogos. A cambio, la investigación antropológica ha documentado diferencias en el ámbito de creencias, valores, prácticas y relaciones sociales (Urban y Koh, 2013).

El análisis de la cultura, entendida como un sistema de interacción de símbolos, que propician la construcción de marcos referenciales para guiar las acciones de los colectivos, es un tejido que ayuda a describir los comportamientos sociales de manera inteligible. Roca i Girona en su trabajo de 2001 apunta que, el análisis cultural desde un abordaje etnográfico, que privilegia la observación participante posibilita, por un lado, y desde una aproximación emic, llevar a cabo una especie de estereovisión bajo la que se recogen las interpretaciones y significados de los acontecimientos y de la conducta de los colectivos, separadamente de las imágenes de los investigadores.

De tal forma que, la combinación de ambas perspectivas permita describir las particularidades del sistema cultural, en la medida en que se incorpore la contradicción y la controversia, así como el consenso. Por otro lado, permite también, la adopción de una perspectiva holística que considera que las conductas y acontecimientos culturales forman parte de sistemas amplios e integrados, donde intervienen factores económicos, políticos, de parentesco, mágicos y religiosos, lo que ayuda a que los investigadores superen las explicaciones simplistas y reduccionistas y a descubrir vínculos entre instituciones locales, regionales, nacionales e internacionales.

Si bien para el estudio de las empresas una de las mayores contribuciones de la antropología se puede ubicar en el análisis de la cultura, para explicar el papel social de los empresarios, la noción de elite es una herramienta indispensable. Hernández considera que, desde la perspectiva analítica antropológica, la elite es una identidad colectiva construida por actores que tienen un proyecto de poder, en una coyuntura particular. Con ello el objeto de análisis es el proceso de producción de la identidad colectiva que un grupo de poder lleva adelante en un contexto sociohistórico específico (Hernández, 2013).

El estudio de las elites plantea entender las relaciones que se establecen en la investigación como conflictivos, negociados continuamente, fluctuantes y dinámicos, por ello la 
etnografía plantea la necesidad de permanecer prolongadamente (Gessaghy, 2011). En la antropología empresarial latinoamericana este ha sido un tema de gran relevancia, en la medida en que las élites empresariales están ligadas a factores coyunturales de la región.

\section{Retos y nuevos aportes de la disciplina: la globalización, el trabajo interdisciplinario y la transferencia de conocimiento.}

El primer gran reto metodológico presente desde los primeros trabajos de antropología y empresa, que se extiende también al análisis de otras sociedades, es la interconexión global que imposibilita aislar los colectivos para su análisis. Desde las últimas décadas del siglo XX, en los trabajos antropológicos se plantea la incidencia de la globalización y del sistema mundial en la construcción y explicación de los problemas sociales de las comunidades que, en primera instancia, podrían parecer locales. En este sentido Azevedo (2012) considera que los antropólogos como el resto de los científicos sociales incorporaron la discusión sobre la dependencia y las perspectivas del sistema mundial que, aunque provienen de campos ajenos a la disciplina, se retoma para explicar el origen y la persistencia del atraso o subdesarrollo económico con el argumento de que aquel es un producto directo del desarrollo del capitalismo.

Por ello, la autora señala que "La gente con la que vivimos y sobre la que escribimos generalmente vive en la periferia 0 en la semi periferia del sistema mundial de Wallerstein. Cuando el precio de sus productos se viene abajo en los mercados mundiales, cuando las corporaciones extranjeras se apoderan de sus tierras o cuando otros inversionistas extranjeros abandonan lo que a una vez fue una región próspera y activa, el centro capitalista influye profundamente en las regiones periféricas y en los pueblos que viven en ellas" (Azevedo, 2012:135).

Considerando lo anterior, el reto de la antropología de la empresa Latinoamericana estriba en enmarcar la perspectiva regional dentro de los sistemas mundiales, pues es la región la que encabeza el proceso de transformación. De manera que, retomar el contexto regional para el análisis de las empresas es un primer acercamiento a sus oportunidades y amenazas en el contexto global. El territorio, visto como fenómeno social, conlleva un orden cultural y social de las acciones que en él se realizan (Azevedo, 2012). Esta vinculación entre lo local y lo global ha sido trabajada profusamente por los antropólogos latinoamericanos en sus estudios sobre empresas y empresarios, pues las acciones de unos y otros solo pueden ser explicadas en un contexto que supera lo que pasa en su localidad o país. Es así como el análisis de lo globlocal se ha visibilizado en la teoría antropológica en parte, gracias a los trabajos sobre empresas y empresarios.

Desde el inicio de los estudios de y sobre empresas los aportes derivados de la antropología, que, como se ha señalado, comienzan con el método etnográfico, también filtran enfoques y conceptos de la disciplina, que se ensamblan a las explicaciones construidas desde otras ciencias sociales. Así, la visión etnográfica en el análisis de la empresa ha completado y corregido significativamente los puntos de vista dominantes que surgen de la economía y del derecho. En los primeros estudios la investigación etnográfica demostró que, al menos internamente, las corporaciones se asemejan a los tipos de sociedades estudiadas durante mucho tiempo por los antropólogos.

Las empresas tienen (desarrollan con el tiempo) sus propias culturas internas distintivas, caracterizadas por la circulación de mitos e historias, prácticas, rituales, creencias y 
valores. Esta noción ha sido retomada por la administración en donde el debate se encuentra en esclarecer el papel que juega la variable cultural al interior de la organización. Los defensores de que la empresa constituye una cultura entienden la organización como una construcción social, edificada simbólicamente y reproducida a través de la interacción social donde la cultura es una metáfora de la organización, en tanto que al igual que ésta, es depositaria y productora de sentido.

Del otro lado del debate, se encuentran los que afirman que la cultura constituye uno de los subsistemas de la organización, una variable más, que debe ser considerada junto a la estructura, el liderazgo, la estrategia o la tecnología (Roca i Girona, 2001). En ambos casos, los análisis antropológicos se suman a los de otras diciplinas para matizar la manera en que las relaciones sociales de la empresa tanto interna como externamente, se construyen y se transforman.

Por último, otro tema en el que los análisis y métodos antropológicos han estado muy involucrados es en la manera en que se transfieren y desarrollan conocimientos y tecnología entre empresas y empresarios, en la medida en que la labor de emprendimiento implica la acumulación de recursos materiales y sociales, pero también, de saberes y conocimientos. La preocupación central de quienes trabajan este tema en Latinoamérica se sitúa en torno al proceso de generación, apropiación y aplicación de nuevo conocimiento en diferentes sectores productivos. Inicialmente, la aproximación a la temática se hizo desde la perspectiva de la gestión y administración de la tecnología, pero bien pronto se entendió que sin considerar los factores socioculturales de la organización que recibe el conocimiento y/o la tecnología, el proceso de asimilación es prácticamente imposible. De ahí que las explicaciones construidas desde quienes se dedican al estudio de la cultura se hacen indispensables, como se muestra en el trabajo sobre una empresa multinacional latinoamericana como Cemex (de Gortari y Santos, 2004).

La literatura construida desde la economía de la innovación señala que, la incorporación de conocimiento y tecnología al sistema productivo, así como el aprendizaje de nuevas formas de producción y distribución de bienes y servicios favorece la competitividad de las empresas. Al mismo tiempo también se reconoce que las formas de aprender, las fuentes de conocimiento utilizadas, los contenidos del aprendizaje varían según las características específicas de las empresas (tamaño, capacidad financiera, competencias tecnológicas acumuladas, esfuerzos activos de aprendizaje, características del proceso de trabajo, etcétera). Sin embargo, en ocasiones pasan por alto que las empresas son actores que se desenvuelven en un contexto histórico, social, político y económico específico, donde las trayectorias laborales y las percepciones (de empresarios, y trabajadores) en torno a los procesos de cambio cobran relevancia singular (Milesi y Motta, 2014).

Es aquí donde los estudios antropológicos han aportado, pero también, la teoría y métodos se han beneficiado de estos trabajos al incorporar variables como la economía y sociedad del conocimiento, las nuevas relaciones culturales construidas a partir de las tecnologías, y muy claramente las de información y comunicación; asuntos que, si bien contribuyen a explicar los procesos de cambio de las empresas, también lo pueden hacer para el actuar de otros colectivos. 


\section{Algunas reflexiones finales}

La revisión sobre los trabajos antropológicos en Latinoamérica, como hemos mostrado a lo largo del artículo, nos permite afirmar que han hecho aportaciones en temas, herramientas, métodos y categorías de análisis para el estudio de la empresa y los empresarios. Quizá la más importante, es que ha permitido ubicar diferencias derivadas de la superposición de referencias culturales del entorno local, regional y global. Lo anterior, a partir del trabajo etnográfico que se lleva a cabo, a través de la observación participante y la perspectiva emic, que supone la descripción densa de la cultura de las empresas y empresarios para entender que recursos y conocimientos se articulan y activan para emprender y, en el caso de la antropología aplicada proporcionar argumentos para comprender y resolver algunos de los problemas que se plantean entre la administración y la fuerza de trabajo, así como con el entorno. Lo anterior, permite resolver los problemas que se presentan con las entrevistas, el levantamiento de la información, así como la postura que debe asumirse frente a los datos obtenidos, lo que posibilita encontrar el modelo de articulación social y su elaboración teórica.

Para entender cómo se fueron construyendo las aportaciones de la antropología para los estudios de la empresa, se resaltan algunos de los hitos por los que ha transcurrido, pero siempre sobre la base de que se trata de una disciplina que incluso desde la tradición de los estudios de las "sociedades simples" ha analizado el entorno social, ideológico y tecnológico, es decir, la cultura de comunidades corporadas y cerradas. Estos aportes han sido recuperados por otras disciplinas, como la psicología y la administración para el análisis de los procesos tecnológicos y las relaciones humanas.

En los estudios antropológicos sobre empresas y empresarios en Latinoamérica, se ha retomado el método comparativo para describir y explicar las similitudes de los entornos industriales locales, enmarcados en los procesos de globalización para analizar cómo sus referencias, inciden en los procesos tecnológicos, estructuras organizacionales y en las culturas empresariales locales, pues muchas de las empresas de la región están insertas en cadenas globales de valor. La antropología de la empresa logra que, en el análisis de sociedades más complejas cercanas a los propios investigadores, se tomen en consideración categorías como la cultura, las redes, el método comparativo, el relativismo cultural y la perspectiva emic.

En América Latina, los estudios sobre empresarios dieron inicio desde el análisis de las elites, a partir de las herramientas de la antropología que ayudan a caracterizar las prácticas y referencias de quienes ocupan posiciones de poder. Igualmente, esta región, tiene una larga tradición en el análisis de la familia que ha estado presente desde los primeros estudios de las empresas. El vínculo entre familia, empresarios y empresa permite entender y explicar los sistemas de parentesco y la manera en que las estructuras y sus relaciones familiares se trasladan a las organizaciones de trabajo. De igual manera, se analiza como las referencias locales de las familias de los empresarios y de los trabajadores, permean en la cultura de las empresas.

En el caso de Latinoamérica, la centralidad de la familia en los estudios sobre las empresas siempre ha tenido un papel importante en los vínculos con la política y el Estado, entre otros. En donde los recursos sociales, materiales, políticos y de conocimientos son claves para que las familias emprendan y mantengan las industrias, talleres y comercios. A los que le siguieron otros, donde se resalta el rol de los empresarios y sus familias, generalmente grandes, para construir y hacer crecer sus empresas; a partir de su inserción en contextos institucionales, 
Familia y empresas un análisis desde la antropología social.

manejo de relaciones políticas, así como de estrategias como las alianzas matrimoniales y prácticas de sucesión. Destaca también el análisis de las redes, categoría utilizada desde la antropología a partir de la década de 1960, que también ha permeado en la región para explicar el emprendimiento.

En suma, en este trabajo se rescatan las aportaciones de la antropología a los estudios de empresa y empresarios en Latinoamérica, las contribuciones metodológicas y teóricas que pueden ser de utilidad para gestionar las diferencias culturales al interior de las empresas y cómo estos estudios han enriquecido el quehacer de la disciplina.

\section{Referencias Bibliográficas}

Azevedo, Rosangela (2012) La agroindustria del limón en el Valle de Tecomán, México: un enfoque antropológico. Iluminuras v.13, n. 30, pp.133-151, jan./jun. Brasil. https://doi.org/10.22456/1984-1191.31126

Baradó, Máximo y Vecchioli, Virginia (2009) Algunos dilemas y desafíos de la antropología de las élites. Etnografías contemporáneas. Argentina. Disponible en: https://www.academia.edu/21481974/_con_Virginia_Vecchioli_Algunos_dilemas_y_d esaf\%C3\%ADos de una antropolog\%C3\%ADa de las elites en Etnograf\%C3\%A Das contempor\%C3\%A1neas A\%C3\%B10 4 Vol 42009 7-20 UNSAM Edita

Basaldúa, Manuel (2005) Los empresarios de la compañía industrial cerillera de san Juan del Rio: Antropología de la industria cerillera. Tesis de doctorado en antropología s/p Universidad Iberoamericana. México

Bueno, Carmen (2016) Configuraciones productivas en la globalización. Trayectorias a la mexicana. Universidad Iberoamericana, CIESAS. México.

Burawoy, Michael (1979) The Anthropology of Industrial work. Annual Review of Anthropology vol 8, Estados Unidos. (Pp 231-266). https://doi.org/10.1146/annurev.an.08.100179.001311

Cárdenas, Julián (2016) Enredando a las elites empresariales en América Latina: Análisis de Interlocking Directorates y propiedad en México, Chile y Brasil. América Latina Hoy, vol. 73. España. (Pp. 15-44). https://www.redalyc.org/pdf/308/30849089002.pdf

Castellani, Ana y Mariana, Heredia (2020) La reproducción fallida de las élites. Inestabilidad y transformaciones de las élites empresariales argentinas entre 1976 y 2015. Revista Española de Sociología no 29 (3). España. (Pp. 467-486). https://doi.org/10.22325/fes/res.2020.30

Cerutti, Mario (2003) Los estudios empresariales en América Latina ¿el debate interminable?. Boletín de Historia Económica, año 1, 2, junio. México. (Pp 3-9). Disponible en: http://www.audhe.org.uy/Boletin_Audhe/Boletin_2/Boletin_02_Cerutti.pdf

COEC (2018) Las mipymes generan el $28 \%$ del PIB en Latinoamérica, pero carecen aún del impulso necesario. Disponible en: https://www.ceoe.es/es/ceoenews/internacional/las-mipymes-generan-el-28-del-pib-en-latinoamerica-perocarecen-aun del\#: :text=En\%20Am\%C3\%A9rica\%20Latina\%2C\%20las\%20mipymes,pol\%C3\%A Dticas\%20p\%C3\%BAblicas\%20de\%20la\%20regi\%C3\%B3n

Cortés, Inés (2013) De la selva y las salinas. Historia social de dos pueblos-empresa en el oriente de Yucatán (1930-1970) Estud. cult. Maya, vol.42. México. (Pp. 119-144). 
Disponible en: http://www.scielo.org.mx/scielo.php?script=sci_abstract\&pid=S0185$25742013000200004 \&$ lng=pt\&nrm=iso

De Gortari, Rebeca y María Josefa, Santos (2004). Learning Paths in Management of Technological Capacity in National Firms in Mexico. Latin American Business, Vol 5, number 2. Reino Unido. (Pp 25-44). https://doi.org/10.1300/J140v05n02_02

De Gortari, Rebeca y María Josefa, Santos (2010) Saberes y parientes en la formación de microempresas rurales: empresarialidad, redes locales y contextos culturales en Marisol Pérez-Lizaur coord. Empresa y familia en México. Universidad Iberoamericana. México.

Durrenberger, Paul (2007) The Anthropology of Organized Labor in the United States. Ann Review of Anthropology, vol 36. Estados Unidos. (Pp 73-88). Disponible en: https://www.annualreviews.org/doi/pdf/10.1146/annurev.anthro.36.081406.094433

Gamst, Frederick (1977) An Integrating View of the Underlying Premises of an Industrial Ethnology in the United States and Canada. Anthropological Quarterly, Jan, Vol. 50, No. 1. Estados Unidos. (Pp. 1-8). https://doi.org/10.2307/3317381

Geertz, Clifford (1987) La interpretación de las culturas. GEDISA, México.

Gessaghi, Victoria (2011) La experiencia etnográfica y la clase alta. ¿Nuevos desafíos para la antropología?. Boletín de Antropología y Educación, 2,3, diciembre. Argentina,

(Pp. 17-26). Disponible en: https://es.scribd.com/document/423538599/Laexperiencia-etnografica-y-la-clase-alta-nuevos-desafios-para-la-antropologia

González, Carmen y Manuel, Basaldúa (2007) La formación de redes sociales en el estudio de actores y familias. Perspectiva de estudio en historia y antropología. Redes. Revista Hispana para el Análisis de Redes Sociales, vol. 12, junio. España. (Pp. 1-27). Disponible en: https://revistes.uab.cat/redes/article/view/v12-n1-gonzalez-basaldua

Hernández, Marcela (2017) Los estudios empresariales en AL: balance y alternativas de análisis" en Jorge Basave y Marcela Hernández (coordinadores), Los estudios de empresarios y empresas una perspectiva internacional. IIEC-UNAM/UAMIztapalapa/Plaza y Valdés. México.

Hernández, Valeria (2013) Genealogía de una elite rural: elucidación antropológica de una práctica de poder. Mundo Agrario, vol. 13, $n^{0} 26$, junio. Argentina. Disponible en: https://www.redalyc.org/pdf/845/84527468004.pdf

Holzberg, Carol y Maureen, Giovannini (1981) Anthropology and Industry reappraisal and new directions. Ann. Review of Anthropology vol 10. Estados Unidos. (Pp. 317-360). https://doi.org/10.1146/annurev.an.10.100181.001533

Jelín, Elizabeth y Paz, Gustavo. (1991) Familia/género en América Latina: Cuestiones históricas contemporáneas. Documento CENDES 69 CENDES, Buenos Aires (Pp 1-33). Disponible en: http://repositorio.cedes.org/handle/123456789/3371

Lima Pedroso de, Arturo (2009) Cuando familia y empresa se vuelven inseparables: hombres de negocios y gestoras familiares Revista Etnografías Contemporáneas. Año 4, número 4, Unsam, Argentina.

Lomnitz, Larissa y Marisol Pérez-Lizaur (1993). Una familia de la élite mexicana, parentesco, clase y cultura 1820-1890. Alianza Editorial. México

Lupton Thomas (1963) On the Shop Floor: Two Studies of Workshop Organization and Output. Oxford Pergamon Press. Reino Unido. 
Milesi, Andrea y Motta, Jorge. (2014) Transformaciones en la mirada empresarial respecto del papel de los trabajadores en empresas autopartistas de Córdoba, Argentina. Antipod. Rev. Antropol. Arqueol. No. 19, mayo-agosto, Colombia, (Pp. 171-188). Disponible en: https://www.redalyc.org/articulo.oa?id=81431457008

Nader, Laura (1972) Up me anthropologist: perspectives gained from studying up, mimeo, 28p. Disponible en: https://files.eric.ed.gov/fulltext/ED065375.pdf

Peña, William (2008) La etnografía, una metodología apropiada al diagnóstico de la responsabilidad social empresarial. Univ. Empresa, vol 7 no 15. Colombia. (Pp. 177183). Disponible en: https://www.redalyc.org/pdf/1872/187214457008.pdf

Pérez-Lizaur, Marisol comp (2010) Empresa y Familia en México, una visión desde la antropología. Universidad Iberoamericana, México.

Ramírez, Luis. (1992) Estratificación, clase y parentesco: empresarios libaneses en el sureste de México. Nueva Antropología, vol XIII no 43. México. (Pp. 123). Disponible en: https://www.redalyc.org/pdf/159/15904311.pdf

Reguera, Andrea (2009) Empresarios de ayer, de hoy y de siempre. Un recorrido latinoamericano por sus formas espacio-temporales América Latina en la historia económica, num 32, jul-dic, México. (Pp. 11). Disponible en: http://www.scielo.org.mx/scielo.php?script=sci_arttext\&pid=S1405-

\section{3\&lng=es\&nrm=iso}

Reygadas Luis (2000) Ensamblando culturas. Diversidad y conflicto en la globalización de la industria. GEDISA, México.

Roca i Girona Jordi (2001) Antropólogos en la empresa?: A propósito de la mal llamada cultura de la empresa". Etnográfica, Vol. 1. Colombia. (Pp. 69-99). Disponible en: http://ceas.iscte.pt/etnografica/docs/vol_05/N1/Vol_v_N1_69-100.pdf

Roca i Girona, Jordi (1999) De la (im)pertienencia del obrero como objeto de estudio de la antropología social. Política y Sociedad, 31, España. (Pp. 201-207). Disponible en: https://revistas.ucm.es/index.php/POSO/article/view/POSO9999230201A

Román, Patricia (2011) Análisis del papel de la familia en la supervivencia de los micronegocios en la ciudad de México. Nueva Antropología, vol. XXIV, núm. 74, enero-junio. México. (Pp. 125-157). Disponible en: http://hdl.handle.net/20.500.11799/38685

Santos, Maria Josefa y de Gortari, Rebeca (coords) (2016) Redes, Herramientas para la competitividad de las empresas rurales en México. IIS UNAM-BUAP- Juan Pablos eds. México.

Urban, Greg. y Kyung-Nan, Koh (2013) Ethnographic research on modern business corporation". Annual Review Anthropology, vol 42. Estados Unidos. (Pp 139-158).: https://doi.org/10.1146/annurev-anthro-092412-155506

White Leslie (1964) La Ciencia de la Cultura, Paidos. Argentina. 